\title{
Gamificación en el ámbito educativo: Un análisis bibliométrico
}

Gamification in the educational field:

A bibliometric analysis

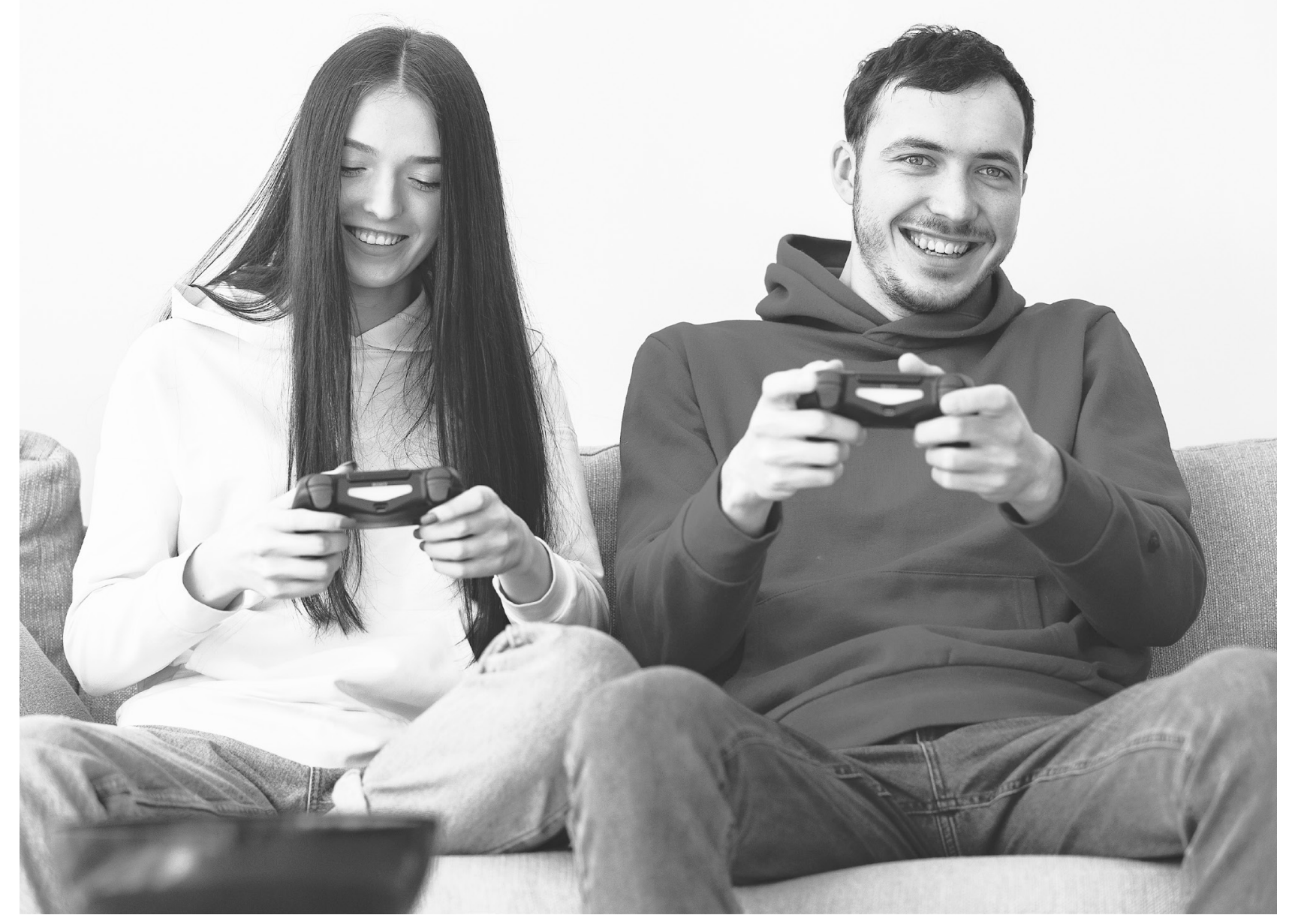




\title{
Gamificación en el ámbito educativo: Un análisis bibliométrico ${ }^{1}$ \\ Gamification in the educational field: A bibliometric analysis
}

\author{
Julieth Katherin Acosta-Medina ${ }^{2}$, Martha Liliana Torres-Barreto ${ }^{3}$, \\ Mileidy Alvarez-Melgarejo ${ }^{4}$, Maira Camila Paba-Medina ${ }^{5}$
}

Artículo recibido en septiembre 16 de 2019; artículo aceptado en octubre 30 de 2019

\begin{abstract}
Este artículo puede compartirse bajo la licencia Licencia Creative Commons Atribución-NoComercial-Compartir Igual 4.0 Internacional y se referencia usando el siguiente formato: Acosta-Medina, J. K., Torres-Barreto, M. L., Alvarez-Melgarejo, M. \& PabaMedina, M. C. (2020). Gamificación en el ámbito educativo: Un análisis bibliométrico. I+D Revista de Investigaciones, 15 (1), $30-39$.

DOI: https://doi.org/10.33304/revinv.v15n1-2020003
\end{abstract}

\section{Resumen}

La gamificación se entiende como la incorporación de elementos propios del juego en contextos que no son lúdicos, con el objetivo de aumentar la concentración y el compromiso, de modo que se alcance una mayor motivación para realizar tareas que a priori pueden parecer poco atractivas. Dada su relevancia, este artículo presenta un análisis bibliométrico acerca del avance que ha tenido el uso de herramientas gamificadas en el ámbito educativo. Para desarrollar esta investigación, se realizó una búsqueda de literatura en Scopus y Web of Science. Los resultados de este ejercicio se analizaron utilizando el software Vantage Point, en términos de tipo de documentos, productividad, análisis léxico y áreas de investigación. Los hallazgos de este estudio indican que la gamificación es una temática cada vez más relevante, lo que se soporta en diferentes investigaciones científicas. Se trata de un campo que puede aplicarse en diversos contextos educativos y que puede generar múltiples beneficios, como aumento de motivación y engagement.

Palabras clave: Análisis bibliométrico, gamificación, educación, motivación, tendencia educativa.

\footnotetext{
Abstract

Gamification is understood as the incorporation of playful elements in contexts that are not recreational, to increase concentration and commitment, in order to increase the motivation to perform tasks that a priori may seem unattractive.

${ }^{1}$ Artículo de revisión de tipo descriptivo, resultado de un proyecto de investigación en curso llamado "DIDACTIC: Plataforma de didáctica motivacional basada en gamificación, como apoyo a los programas de formación virtual de la región de Antioquia", financiado por Colciencias, perteneciente al área de ciencias sociales, subárea de educación, desarrollado en el grupo de investigación Finance and Management de la Universidad Industrial de Santander (Bucaramanga, Colombia): Dirección: carr.a 27 n. ${ }^{\circ}$ 9. PBX: 6344000. Fecha de inicio: 27 de junio de 2019.

${ }^{2}$ Ingeniera industrial vinculada a la Universidad Industrial de Santander (Bucaramanga, Colombia). Dirección: carr. 27 n. ${ }^{\circ} 9$. PBX: 6344000. ORCID ID: https://orcid.org/0000-0001-6910-069X. Correo electrónico: katheacosta19@gmail.com.

${ }^{3}$ Doctora en Ciencias Económicas. Docente e investigadora del grupo de Investigación Finance and Management. Universidad Industrial de Santander (Bucaramanga, Colombia). Dirección: carr.a 27 n. 9. 9BX: 6344000. ORCID ID: http://orcid.org/0000-0002-4388-5991. Correo electrónico institucional: mltorres@uis.edu.co.

${ }^{4}$ Administradora de Empresas. Asesora externa vinculada a la Universidad Industrial de Santander (Bucaramanga, Colombia). Dirección: carr.a 27 n. ${ }^{\circ}$ 9. PBX: 6344000. ORCID ID: http://orcid.org/0000-0003-1752-8023. Correo electrónico: mileidyalvarezm@gmail.com.

${ }^{5}$ Estudiante de Ingeniería Industrial de la Universidad Industrial de Santander (Bucaramanga, Colombia). Dirección: carr.a 27 n. ${ }^{\circ} 9$. PBX: 6344000. ORCID ID: https://orcid.org/0000-0002-0453-9367. Correo electrónico: mairacami@hotmail.com.
} 
Given its relevance, this article presents a bibliometric analysis of the progress made by the use of gamified tools in the educational field. For the development of this research, a search of literature was conducted in Scopus and Web of Science. The results of this exercise were analyzed using Software Vantage Point, in terms of the type of documents, productivity, lexical analysis, and research areas. The findings of this study indicate that gamification is an increasingly relevant subject supported by different scientific research. It is a field that can be applied in various educational contexts and that can generate multiple benefits such as increased motivation and engagement.

Keywords: Bibliometric analysis, gamification, education, motivation, educational trend.

\section{Introducción}

La educación tiene gran importancia para el desarrollo del ser humano y de la sociedad, ya que facilita desplegar las capacidades de las personas y promueve el crecimiento económico y el bienestar social. Sin embargo, las prácticas educativas no permanecen estáticas. Las herramientas pedagógicas, técnicas y metodologías evolucionan constantemente.

En consecuencia, el uso de las tecnologías de información y comunicación (TIC) en los entornos educativos cada día es más generalizado. Estudios científicos han demostrado que la aplicación de tecnologías combinadas con recursos multimedia han permitido a los estudiantes aumentar su compresión de diferentes conceptos (Simanca, Abuchar, Blanco \& Carreño, 2017). Adicionalmente, se observa que la enseñanza a través de entornos virtuales de aprendizaje se ha convertido en una tendencia que muchas instituciones educativas están incorporando en sus políticas de desarrollo y prospectiva académica.

En este contexto, el desarrollo de la gamificación -también Ilamada 'ludificación'- puede verse como un ejemplo de la continua renovación de la práctica educativa. Se trata de una de las tendencias de innovación educativa que se puede usar para soportar el proceso de enseñanza y aprendizaje, al ayudar tanto a los maestros, en el plano de mejorar el proceso de seguimiento, como a los estudiantes, en el de aumentar su motivación para aprender de nuevas maneras y disfrutar sus actividades académicas.
Por esto, en los últimos años estas herramientas gamificadas han sido aplicadas en algunas instituciones de educación en diferentes fases de formación educativa (básica, secundaria, superior), así como en distintas modalidades de estudio (presencial, semipresencial y virtual) (Kasurinen \& Knutas, 2018).

Basado en lo anterior, el presente análisis bibliométrico tiene como propósito analizar los avances en el uso de las herramientas didácticas gamificadas en el ámbito educativo, a partir de lo encontrado en la literatura.

Este documento comienza con la descripción de la gamificación, sus tipos y elementos clave. Seguido de esto, se presentan los resultados encontrados en cuanto a número y tipo de documentos, palabras clave, revistas y países con mayor número de publicaciones, áreas de investigación y análisis de citación. Finalmente se presentan las conclusiones del estudio.

\section{Gamificación}

La gamificación hace referencia al uso de elementos propios de los juegos en contextos no lúdicos (Deterding, Dixon, Khaled \& Nacke, 2011). La incorporación de estos elementos genera en el cerebro humano la liberación de dopamina, lo que produce en los individuos sentimientos positivos de diversión, motivación y atención. Esto facilita el reconocimiento y procesamiento de nuevo conocimiento; es decir, promueve el aprendizaje (ver Figura 1) (LlorensLargo et al., 2016; Torres-Menárquez, 2016).

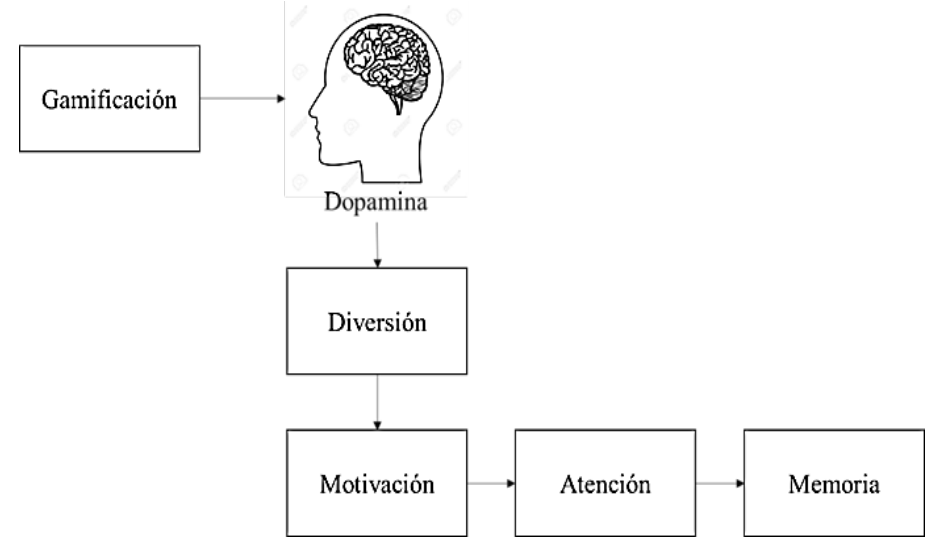

Figura 1. Consecuencias de la gamificación en el cerebro humano. Fuente: Adaptado de Llorens-Largo et al. (2016) y Torres-Menárquez (2016). 
Es importante no confundir el concepto de gamificación con otros términos similares (ver Figura 2), como el de juegos, que constituyen actividades lúdicas cuyo principal objetivo es la diversión (como el ajedrez); o el de juegos serios, que constituyen juegos en sí mismos y están diseñados con objetivos formativos concretos, que van más allá del simple entretenimiento (como un simulador de vuelo que tiene como objetivo enseñar a los pilotos a reaccionar ante distintas situaciones). La gamificación no es un juego propiamente dicho, sino una herramienta que aplica elementos de juegos en ámbitos reales, con el objetivo de motivar a los individuos a realizar tareas que a priori pueden parecer poco atractivas (Deterding et al., 2011; Marczewski, 2013).

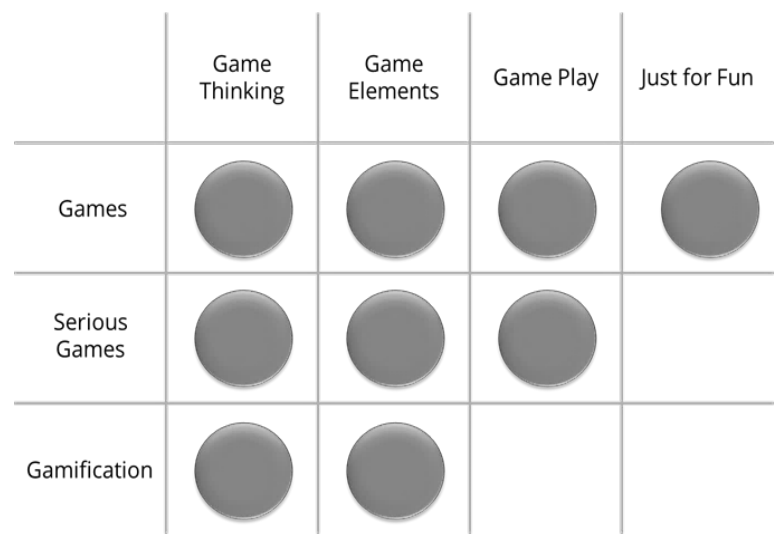

Figura 2. Términos similares a gamificación. Fuente: Adaptado de Marczewski (2013).

\section{Tipos de gamificación}

Existen dos formas de incorporar la gamificación en los contextos educativos: la gamificación superficial o de contenido, que se utiliza de forma puntual en una clase o actividad; y la gamificación estructural o profunda, que se implementa en toda la estructura de un curso (Garone \& Nesteriuk, 2019).

\section{Elementos clave de las herramientas gamificadas}

De acuerdo con el modelo planteado por Werbach y Hunter, (2012), las herramientas gamificadas deben tener tres elementos principales: dinámica, mecánica y componentes (ver Figura 3).

Los componentes son los recursos que se utilizan para diseñar la actividad en la práctica de la gamificación. Por ejemplo, avatares, puntos, insignias, niveles, misiones, barras de progreso y tablas de clasificación. Por su parte, la mecánica hace referencia a las reglas o normas de funcionamiento de la herramienta. Estas pueden ser de varios tipos: competición, colección, cooperación, equilibrio o desafíos. Dicha mecánica se pone en marcha gracias a la dinámica, es decir, a los contextos en los que se desarrolla la gamificación que corresponden a narrativa, emociones, progresión, restricciones y relaciones sociales (Werbach \& Hunter, 2012).

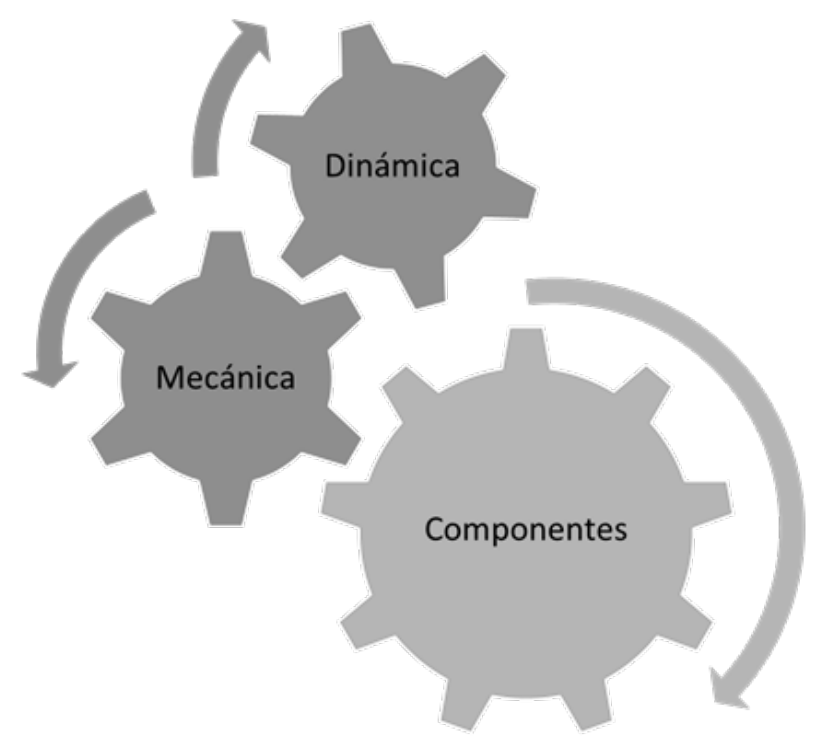

Figura 3. Elementos de las herramientas gamificadas. Fuente: Adaptado de Werbach y Hunter (2012).

\section{Tipos de jugadores en herramientas gamificadas}

Al implementar la gamificación, hay que tener en cuenta que existen diferentes tipos de participantes de acuerdo con sus intereses y motivaciones. Según Marczewski, (2013) los usuarios de la gamificación se clasifican en:

- Exploradores: Les gusta ver todas las posibilidades de la herramienta y realizar varios intentos. Prefieren sentirse parte de una historia y ser un personaje.

- Socializadores: Su objetivo principal es la interacción social, por lo que mantienen conversaciones con los demás participantes y trabajan de forma colaborativa.

- Pensadores: Intentan diferentes estrategias para resolver problemas hasta encontrar una solución, por lo que constantemente prueban su conocimiento.

- Filántropos: Se enfocan en ayudar a los demás con lo que necesitan, sin esperar nada a cambio.

- Triunfadores: Tienen el deseo constante de ganar, superar todos los retos, cumplir todas las misiones y avanzar a otros niveles.

- Revolucionarios: Son la población más pequeña dentro de los tipos de participantes. Les interesa conocer lo más extraño. Para ellos, ganar no es suficiente; disfrutan los reconocimientos y están motivados por vencer al sistema. 


\section{Metodología}

\section{Tipo de estudio}

Este estudio es de tipo descriptivo y busca estudiar los avances en el uso de las herramientas gamificadas en los entornos educativos, a partir del análisis de contenido cuantitativo originado de una búsqueda de literatura. La investigación es no experimental, porque valora algunas características de la documentación científica revisada y analiza la trazabilidad de la literatura desde los orígenes de la gamificación.

\section{Procedimiento}

Para el presente artículo se realizó una búsqueda de literatura enfocada en los avances relacionados con el uso de las herramientas didácticas motivacionales en el ámbito educativo y basadas en la gamificación. Para este ejercicio, se usó el portal de búsqueda Scopus y la base de datos multidisciplinaria ISI Web of Science, en los que se revisaron todos los años disponibles en cada base de datos (1980-2019 para Web of Science, y 1976-2019 para Scopus), sin restricción de tipo de documento, área de investigación o idioma.

Adicionalmente, para complementar la información se realizó una búsqueda basada en el principio de bola de nieve; es decir, se revisaron aquellas referencias bibliográficas citadas en la literatura, consultadas e importantes para este estudio. Posteriormente, del total de documentos encontrados (420) se escogieron los de mayor afinidad y pertinencia al tema de interés (60), teniendo en cuenta que el presente estudio se enmarca en el proyecto "DIDACTIC: Plataforma de didáctica motivacional basada en gamificación, como apoyo a los programas de formación virtual de la Región de Antioquia". Finalmente, la documentación elegida se analizó utilizando el software Vantage Point.

\section{Resultados}

En esta sección se presentan los hallazgos encontrados en el análisis en cuanto a productividad anual, tipos de documentos analizados, análisis léxico de las palabras clave y de los títulos, revistas y países con mayor número de publicaciones, áreas de investigación y análisis de citación.

\section{Artículos por año}

Gamificación es un término originado en la industria de los medios digitales. El primer uso documentado de este término data del 2008 (Paharia, 2010), pero tuvo una adopción generalizada antes de la segunda mitad del 2010 (Deterding et al., 2011). Al analizar los 60 documentos científicos de mayor afinidad y pertinencia a la temática de investigación, tal como se observa en la Figura 4, se evidencia una tendencia creciente desde
2015 en el número de publicaciones. Incluso, aunque no ha terminado el año 2019 (momento en el que se realiza la búsqueda), existen 12 documentos publicados, un número similar a las publicaciones del año anterior.

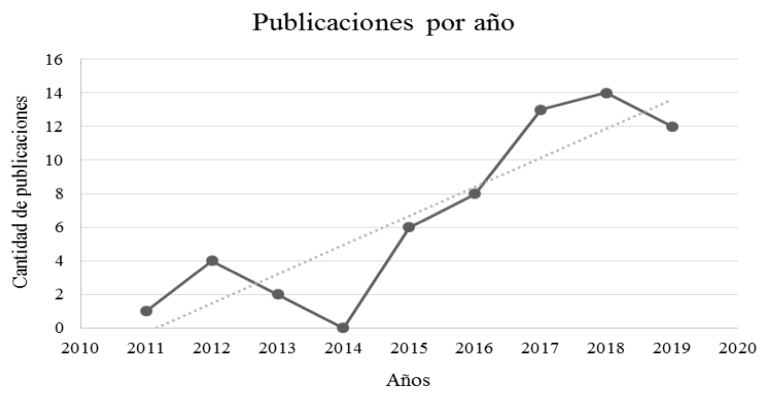

Figura 4. Publicaciones por año. Fuente: Autoras utilizando el software Vantage Point.

\section{Tipos de documentos}

En cuanto al tipo de documentos analizados, el $65 \%$ corresponde a artículos; el $25 \%$, a actas -o conference proceedings, en inglés-, y el 10\%, a capítulos de libros (ver Figura 5). Estas publicaciones están principalmente en idioma inglés $(85 \%)$, y en menor medida en otras lenguas, como español (8\%), portugués $(5 \%)$ y alemán $(2 \%)$.

Tipo de Documento

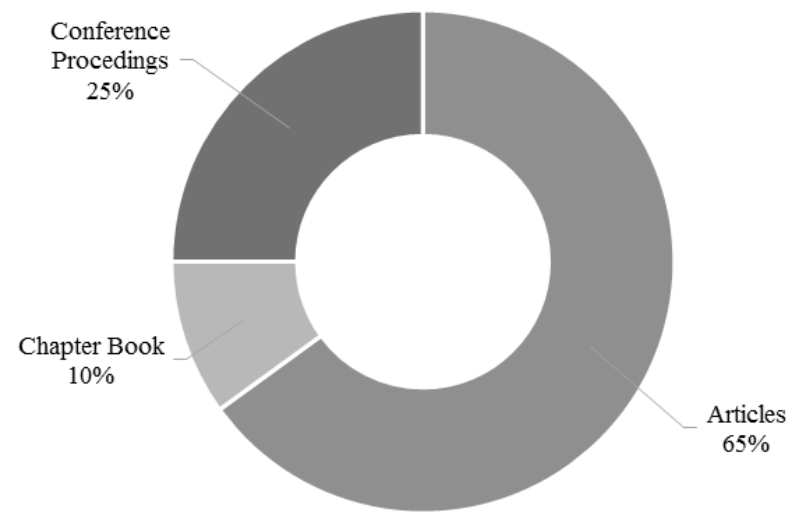

Figura 5. Tipo de documento. Fuente: Autoras utilizando el software Vantage Point.

\section{Análisis léxico basado en los títulos}

A partir de la extracción de palabras de los títulos, se identifica una clara relación de la gamificación con los elementos de los juegos, debido a la definición misma de gamificación. Además, se muestra una fuerte conexión con términos como educación, aprendizaje, estudiantes, educación virtual, innovación y motivación (ver Figura 6). Esto se debe a que la mayoría de los estudios se centran en el desarrollo de proyectos innovadores multidisciplinarios de gamificación que involucran varios cursos bajo plataformas de aprendizaje electrónico. 


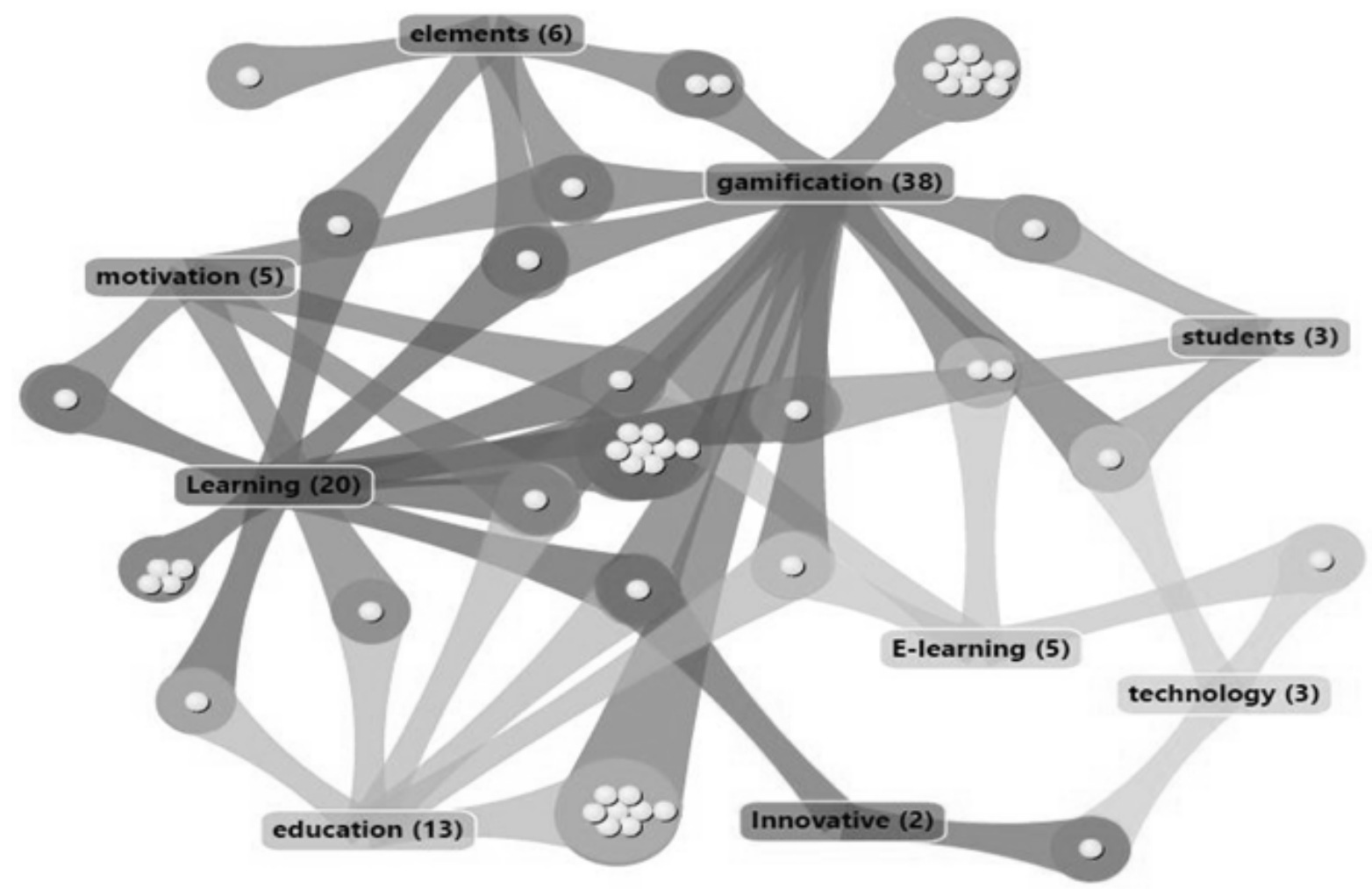

Figura 6. Cluster map de principales palabras de los títulos. Fuente: Autoras utilizando el software Vantage Point.

\section{Análisis léxico de las palabras clave}

Al analizar las palabras clave de los 60 documentos estudiados se encuentra que los términos con mayor frecuencia son gamification, education, teaching and learning, online course, e-learning (ver Figura 7). Adicionalmente, palabras clave como motivation y engagement indican los beneficios de la gamificación. Cabe resaltar que estos hallazgos son similares a los encontrados por Taspinar, Schmidt y Schuhbauer (2016), quienes encuentran en sus investigaciones que al incorporar herramientas gamificadas en ambientes educativos aumentan la motivación y el compromiso de los estudiantes.

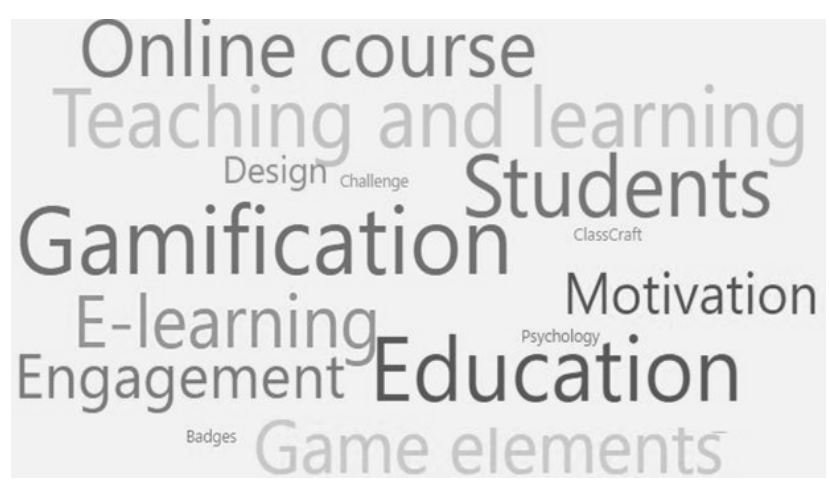

Figura 7. Nube de palabras clave. Fuente: Autoras utilizando el software Vantage Point.

\section{Revistas con más productividad}

En la Tabla 1 se presentan las revistas que tienen el mayor número de publicaciones, junto con su índice de impacto normalizado (SNIP), un factor de medición basado en la comparación de publicaciones dentro de sus campos temáticos que se calcula contabilizando la frecuencia con la que los autores citan otros documentos y la inmediatez del impacto de dicha cita. De estas, destacan Computer Science Review y Computers in Human Behavior, revistas académicas dedicadas a investigaciones relevantes para la informática, lo que refleja la incorporación de herramientas gamificadas soportadas en tecnologías de la información y comunicación (TIC).

\section{Áreas de investigación}

La mayoría de los estudios analizados se realizaron en las áreas de educación (35\%) y ciencias de la computación $(30 \%)$. También hay otras áreas relacionadas, como ingeniería $(8 \%)$, ciencias sociales $(8 \%)$, psicología $(7 \%)$, matemáticas $(6 \%)$, economía $(5 \%)$ y salud $(1 \%)$ (ver Figura 8). Estos resultados confirman lo encontrado por Dicheva, Dichev, Agre y Angelova (2015), y Seaborn y Fels (2015), quienes afirman que la gamificación es un campo multidisciplinario que puede incorporarse en los procesos de enseñanza y aprendizaje de múltiples áreas. 
Revistas con mayor número de publicaciones

\begin{tabular}{cccc}
\hline Ranking & Número de publicaciones & Revista & SNIP 2018 \\
\hline 1 & 4 & Computer Science Review & 5.391 \\
2 & 4 & Computers in Human Behavior & 2.245 \\
3 & 4 & ACM International Conference Proceeding Series & 0.594 \\
4 & 2 & Computers and Education & 3.797 \\
5 & 2 & Electronic Journal of E-Learning & 1.454 \\
6 & 2 & International Journal of Game- Based Learning & 1.430 \\
7 & 2 & International Journal of Emerging Technologies in Learning & 1.388 \\
8 & 2 & International Journal of Innovation and Learning & 1.015 \\
\hline
\end{tabular}

Fuente: Autoras.

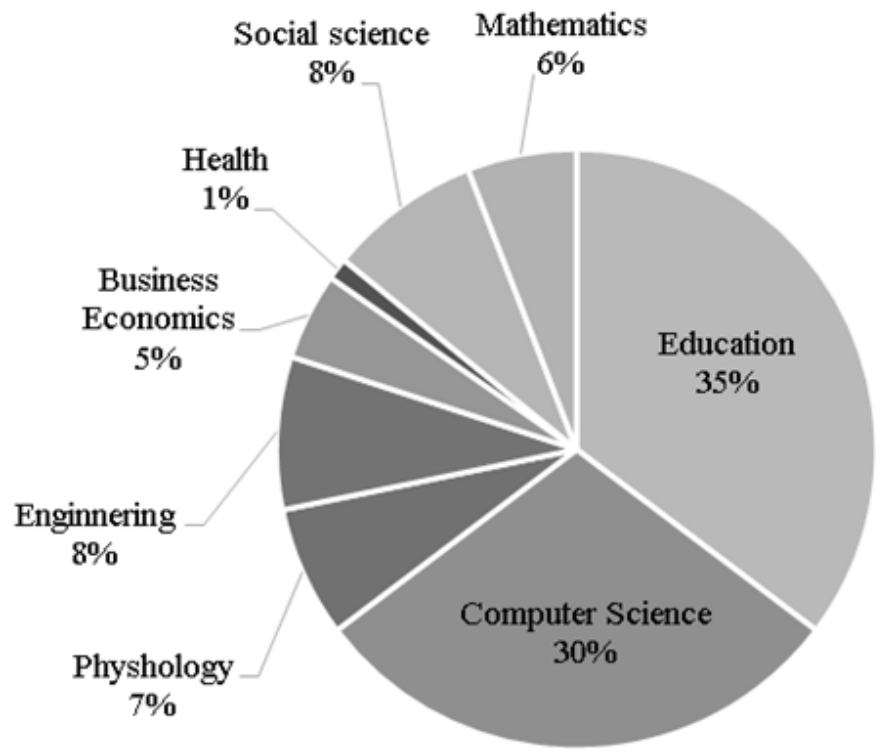

Figura 8. Áreas de investigación. Fuente: Autoras utilizando el software Vantage Point.

\section{Países con mayor número de publicaciones}

Al analizar la productividad por países, tal como se observa en la Figura 9, se comprueba que Estados Unidos y España son los países con el mayor número de publicaciones en este tópico ( 11 y 7 , respectivamente).

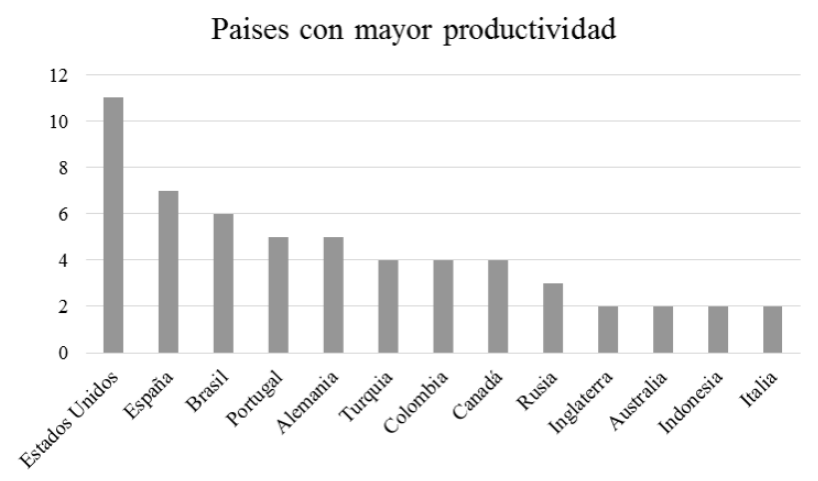

Figura 9. Países con mayor número de publicaciones. Fuente: Autoras utilizando el software Vantage Point.
Por su parte, Colombia tiene 4 investigaciones publicadas (3 realizadas en la región de Antioquia). Estos estudios abordan la implementación de herramientas gamificadas en entornos educativos virtuales, y sus hallazgos indican los beneficios de la gamificación con relación al aumento de la motivación de los estudiantes (Gasca-Hurtado, Gomez \& Zepeda, 2018; Gonzalez, Gomez \& Echeverri, 2017); al incremento de la participación y el compromiso (Pascuas, Vargas \& Muñoz, 2017); a la disminución de la deserción estudiantil, y al crecimiento en la curva de aprendizaje (Melo-Solarte \& Díaz, 2018).

\section{Análisis de citación}

El documento más relevante ha recibido 2049 citaciones. Fue realizado por Deterding et al. (2011) y se publicó en Estados Unidos, pero se presentó en Finlandia, en la Conferencia MindTrek. Esta acta se titula "From game design elements to gamefulness: Defining gamification", e investiga los orígenes históricos de la gamificación. Deterding et al. (2011) aportan la definición actual de 
gamificación y sugieren incorporar estrategias de esta índole, debido a que son un fenómeno novedoso y lúdico que posee gran efectividad.

Así mismo, cabe resaltar el artículo titulado "Gamification in theory and action: A survey", escrito por Seaborn y
Fels (2015), que ha recibido 299 citaciones. Se publicó en Inglaterra y aborda conceptos teóricos de gamificación, comparaciones con enfoques relacionados y hallazgos de su aplicación en diversos contextos. El total de publicaciones que tienen más de 10 citaciones se muestra en la Tabla 2.

Tabla 2

Publicaciones más citadas

\begin{tabular}{|c|c|c|c|}
\hline Ranking & Frecuencia & Autores & Título \\
\hline 1 & 2049 & (Deterding et al., 2011) & $\begin{array}{c}\text { "From game design elements to gamefulness: Defining" } \\
\text { 'gamification"” }\end{array}$ \\
\hline 2 & 299 & (Seaborn \& Fels, 2015) & "Gamification in theory and action: A survey" \\
\hline 3 & 190 & (Dicheva et al., 2015) & "Gamification in education: A systematic mapping study" \\
\hline 4 & 114 & (Li, Grossman \& Fitzmaurice, 2012) & $\begin{array}{l}\text { "GamiCAD: A gamified tutorial system for first time AutoCAD } \\
\text { users" }\end{array}$ \\
\hline 5 & 80 & (Sailer, Hense, Mayr \& Mandl, 2017) & $\begin{array}{c}\text { "How gamification motivates: An experimental study of the } \\
\text { effects of specific game design elements on psychological } \\
\text { need satisfaction" }\end{array}$ \\
\hline 6 & 67 & $\begin{array}{l}\text { (Mekler, Brühlmann, Tuch \& Opwis, } \\
\text { 2017) }\end{array}$ & $\begin{array}{l}\text { "Towards understanding the effects of individual gamification } \\
\text { elements on intrinsic motivation and performance" }\end{array}$ \\
\hline 7 & 55 & $\begin{array}{l}\text { (De-Marcos, Garcia-Lopez \& Garcia- } \\
\text { Cabot, 2016) }\end{array}$ & $\begin{array}{c}\text { "On the effectiveness of game-like and social approaches in } \\
\text { learning: Comparing educational gaming, gamification \& socia } \\
\text { networking" }\end{array}$ \\
\hline 8 & 41 & $\begin{array}{l}\text { (McDaniel, Lindgren \& Friskics, } \\
\text { 2012) }\end{array}$ & $\begin{array}{c}\text { "Using badges for shaping interactions in online learning } \\
\text { environments" }\end{array}$ \\
\hline 9 & 21 & $\begin{array}{l}\text { (Borras-Gene, Martiñez-Nuñez \& } \\
\text { Fidalgo-Blanco, 2016) }\end{array}$ & $\begin{array}{l}\text { "New challenges for the motivation and learning in } \\
\text { engineering education using gamification in } \mathrm{MOOC}^{\prime}\end{array}$ \\
\hline 10 & 19 & (Yildirim, 2017) & $\begin{array}{l}\text { "The effects of gamification-based teaching practices on } \\
\text { student achievement and students' attitudes toward lessons" }\end{array}$ \\
\hline 11 & 19 & $\begin{array}{l}\text { (Osipov, Nikulchev, Volinsky \& } \\
\text { Prasikova, 2015) }\end{array}$ & $\begin{array}{c}\text { "Study of gamification effectiveness in online e-learning } \\
\text { systems" }\end{array}$ \\
\hline 12 & 19 & (Olsson, Mozelius \& Collin, 2015) & $\begin{array}{c}\text { "Visualisation and gamification of e-learning and } \\
\text { programming Education" }\end{array}$ \\
\hline 13 & 14 & (Kyewski \& Krämer, 2018) & $\begin{array}{l}\text { "To gamify or not to gamify? An experimental field study of the } \\
\text { influence of badges on motivation, activity, and performance } \\
\text { in an online learning course" }\end{array}$ \\
\hline 14 & 12 & (Kasurinen \& Knutas, 2018) & $\begin{array}{l}\text { "Publication trends in gamification: A systematic mapping } \\
\text { study" }\end{array}$ \\
\hline 15 & 11 & (Aldemir, Celik \& Kaplan, 2018) & $\begin{array}{c}\text { "A qualitative investigation of student perceptions of game } \\
\text { elements in a gamified course" }\end{array}$ \\
\hline 16 & 11 & (Llorens-Largo et al., 2016) & "Gamification of the learning process: Lessons learned" \\
\hline
\end{tabular}

Fuente: Autoras.

\section{Conclusiones}

Los hallazgos del presente estudio indican que la gamificación se ha convertido en un tema con creciente interés para investigaciones científicas y en una herramienta con gran potencial para aplicarse en múltiples contextos educativos.

Cabe resaltar que, aunque la mayoría de los documentos analizados corresponden a artículos científicos, existe gran cantidad de publicaciones que corresponden a actas -o conferences proceedings-, lo que refleja el número considerable de investigaciones en el campo de la gamificación que se presentan en distintos eventos académicos, como congresos o conferencias. Asimismo,
Estados Unidos destaca como el país líder en la investigación de la gamificación; además, el documento más citado fue publicado en este país y corresponde a un acta.

En cuanto a las revistas, existe un número importante de ellas que publican investigaciones relacionadas con la gamificación en la educación. Por tal motivo, en este estudio no existe una revista académica que resalte por su cantidad de publicaciones. La mayoría de estas pertenecen principalmente a las áreas de ciencias de la computación y ciencias sociales.

Además, el análisis bibliométrico permitió identificar los beneficios de la gamificación, de entre los cuales los 
más significativos son el aumento de la motivación, la participación y el compromiso, así como la disminución de la deserción estudiantil. Lo anterior resulta un buen argumento para la aplicación de herramientas gamificadas en las diferentes modalidades educativas.

Para futuras investigaciones se recomienda tener en cuenta bases de datos adicionales, como EBSCOhost, ProQuest y PubMed, ya que pueden poseer información relevante acerca de la gamificación. También se sugiere incorporar documentación proveniente de la literatura gris y las disertaciones, con el fin de tener información más amplia sobre la temática investigada.

\section{Agradecimientos}

Agradecimiento especial, por el apoyo financiero recibido para la realización de este proyecto, a la Universidad Industrial de Santander y al Sistema General de Regalías, fondos de CTel, de la Gobernación de Antioquia, administrados a través del Patrimonio Autónomo Fondo Nacional de Financiamiento para la Ciencia, la Tecnología y la Innovación Francisco José de Caldas (Colciencias).

\section{Referencias}

Aldemir, T., Celik, B., \& Kaplan, G. (2018). A qualitative investigation of student perceptions of game elements in a gamified course. Computers in Human Behavior, 78, 235-254. https://doi.org/10.1016/j. chb.2017.10.001

Borras-Gene, O., Martiñez-Nuñez, M., \& Fidalgo-Blanco, Á. (2016). New Challenges for the motivation and learning in engineering education using gamification in MOOC. International Journal of Engineering Education, 32(1), 501-512.

De-Marcos, L., Garcia-Lopez, E., \& Garcia-Cabot, A. (2016). On the effectiveness of game-like and social approaches in learning: Comparing educational gaming, gamification and social networking. Computers \& Education, 95, 99113. https://doi.org/10.1016/j.compedu.2015.12.008

Deterding, S., Dixon, D., Khaled, R., \& Nacke, L. (2011). From game design elements to gamefulness. In Proceedings of the 15th International Academic MindTrek Conference on Envisioning Future Media Environments - MindTrek'11 (p. 9). New York, New York, USA: ACM Press. https://doi. org/10.1145/2181037.2181040

Dicheva, D., Dichev, C., Agre, G., \& Angelova, G. (2015). Gamification in Education: A Systematic Mapping Study. Educational Technology \& Society, 18(3), 75-88.

Garone, P., \& Nesteriuk, S. (2019). Gamification and Learning: A Comparative Study of Design Frameworks. In 10th International Conference on Digital Human Modeling and Applications in Health, Safety, Ergonomics and Risk Management (pp. 473-487). https://doi. org/10.1007/978-3-030-22219-2_35

Gasca-Hurtado, G. P., Gomez, M. C., \& Zepeda, V. V.
(2018). Gamification experience of an educational environment in software engineening: Gamifying a course of agility for software process improvement. In 2018 13th lberian Conference on Information Systems and Technologies (CISTI) (pp. 1-6). IEEE. https://doi. org/10.23919/CISTI.2018.8399233

González, L., Gomez, M. C., \& Echeverri, J. A. (2017). Motivation and Virtual Education in Computer Science: Case Universidad de Medellín-Colombia. IEEE Latin America Transactions, 15(6), 1176-1181. https:// doi.org/10.1109/TLA.2017.7932706

Kasurinen, J., \& Knutas, A. (2018). Publication trends in gamification: A systematic mapping study. Computer Science Review, 27, 33-44. https://doi.org/10.1016/j. cosrev.2017.10.003

Kyewski, E., \& Krämer, N. C. (2018). To gamify or not to gamify? An experimental field study of the influence of badges on motivation, activity, and performance in an online learning course. Computers and Education, 118(April 2017), 25-37. https://doi.org/10.1016/j. compedu.2017.11.006

Li, W., Grossman, T., \& Fitzmaurice, G. (2012). GamiCAD. In Proceedings of the 25th annual ACM symposium on User interface software and technology - UIST '12 ( $p$. 103). New York, New York, USA: ACM Press. https:// doi.org/10.1145/2380116.2380131

Llorens-Largo, F., Gallego-Duran, F. J., Villagra-Arnedo, C. J., Compan-Rosique, P., Satorre-Cuerda, R., \& MolinaCarmona, R. (2016). Gamification of the Learning Process: Lessons Learned. IEEE Revista lberoamericana de Tecnologias Del Aprendizaje, 11(4), 227-234. https://doi.org/10.1109/RITA.2016.2619138

Marczewski, A. (2013). Gamification: A Simple Introduction. McDaniel, R., Lindgren, R., \& Friskics, J. (2012). Using badges for shaping interactions in online learning environments. In 2012 IEEE International Professional Communication Conference (pp. 1-4). IEEE. https:// doi.org/10.1109/IPCC.2012.6408619

Mekler, E. D., Brühlmann, F., Tuch, A. N., \& Opwis, K. (2017). Towards understanding the effects of individual gamification elements on intrinsic motivation and performance. Computers in Human Behavior, 71, 525-534. https://doi.org/10.1016/j.chb.2015.08.048

Melo-Solarte, D. S., \& Díaz, P. A. (2018). El Aprendizaje Afectivo y la Gamificación en Escenarios de Educación Virtual. Información Tecnológica, 29(3), 237-248. https://doi.org/10.4067/S0718-07642018000300237

Olsson, M., Mozelius, P., \& Collin, J. (2015). Visualisation and Gamification of e-Learning and Programming Education. The Electronic Journal of E-Learning, 13(6), 441-454. Recuperado de www.ejel.org

Paharia, R. (2010). Who coined the term "gamification"? September 5, 2019. Recuperado de http://goo.gl/ CvcMs

Pascuas, Y., Vargas, E., \& Muñoz, J. (2017). Experiencias motivacionales gamificadas: una revisión sistemática de literatura. Innovación Educativa, 17(75), 63-80. 
Sailer, M., Hense, J. U., Mayr, S. K., \& Mandl, H. (2017). How gamification motivates: An experimental study of the effects of specific game design elements on psychological need satisfaction. Computers in Human Behavior, 69, 371-380. https://doi.org/10.1016/j. chb.2016.12.033

Seaborn, K., \& Fels, D. I. (2015). Gamification in theory and action: A survey. International Journal of HumanComputer Studies, 74, 14-31. https://doi.org/10.1016/j. ijhcs.2014.09.006

Simanca, F., Abuchar, A., Blanco, F., \& Carreño, P. (2017). Implementación de herramientas tecnológicas en los procesos de enseñanza aprendizaje de los triángulos. I+D Revista de Investigaciones, 10(2), 71-79.

Taspinar, B., Schmidt, W., \& Schuhbauer, H. (2016). Gamification in Education: A Board Game Approach to Knowledge Acquisition. Procedia Computer Science, 99, 101-116. https://doi.org/10.1016/j.procs.2016.09.104

Torres-Menárquez, A. (2016, July 7). El cerebro necesita emocionarse para aprender. El País Periódico Global.

Osipov, I. V., Nikulchev, E., Volinsky, A. A., \& Prasikova, A. Y. (2015). Study of Gamification Effectiveness in Online e-Learning Systems. International Journal of Advanced Computer Science and Applications, 6(2). https://doi. org/10.14569/ijacsa.2015.060211

Werbach, K., \& Hunter, D. (2012). For the Win: How Game Thinking Can Revolutionize Your Business. Wharton Digital Press.

Yildirim, I. (2017). The effects of gamification-based teaching practices on student achievement and students' attitudes toward lessons. The Internet and Higher Education, 33, 86-92. https://doi. org/10.1016/j.iheduc.2017.02.002 